\title{
Evaluation of Commercial Connectors for Active Neural Implants
}

\author{
Henry T. Lancashire, Maryam Habibollahi, Dai Jiang, and Andreas Demosthenous
}

\begin{abstract}
Multichannel connectors enable part-replacement of implanted active neural interfaces. For pre-clinical investigation, commercially available miniature connectors enable high channel counts with reduced size and cost. In this paper, Omnetics Nano Circular connectors were encapsulated with medical grade silicone, and assembled using an approach proposed used in surgery. Three 11-pin connectors were tested in PBS for 336 days with cyclic loading for a total of 66 days. A single connector failed with current leakage between channels due to moisture at the connecting interface, and with corrosion at 3 solder joints. The surviving connectors maintained a low contact impedance and high between-channel impedance over 336 days. Inspection of the failed sample emphasizes the need for stress relief near implanted connectors and void-free encapsulation.
\end{abstract}

\section{INTRODUCTION}

Modular implantable neural interfaces allow the replacement of part of failed or damaged systems. For example, implantable deep brain stimulator pulse generators can be replaced without disrupting the brain-implant interface. To facilitate part-replacement of a device implantable electrical connectors are required, either in-line on connecting cables, or mounted on the active device [1].

For advanced devices connector requirements include: high channel count; minimal size; low contact impedance; good isolation between channels; biocompatibility; and stability in the body environment [2]. There is a particular trade-off between connector size and channel count. Neural electrode designs with 10's to 100's of electrode sites are common, while long term implantable connectors typically have fewer than 10 channels [1], [3], [4].

A further consideration for pre-clinical investigation is cost. Using a single multichannel connector in place of multiple low-channel-count connectors will reduce the costs at design and assembly, and reduce surgical time. The connector mating surfaces, and connections to any cables or implants, must be protected from the moist, corrosive, environment of the body. Protection methods for implantable connectors include silicone encapsulation [4], epoxy encapsulation [5], acrylic embedding (as part of a head mounting) [6], compression sleeves [7], and metal-in-ceramic hermetic feedthroughs [8].

Commercially available Omnetics Nano Circular (NC) connectors provide up to 39 contacts at a density of 0.8 channels per $\mathrm{mm}^{2}$ cross sectional area [9]. Omnetics connectors are not designed, or demonstrated to be suitable,

*Research supported by the European Commission under H2020EU.1.2.2. - FET Proactive (Agreement ID: 824071; project NeuHeart), and the Engineering and Physical Sciences Research Council (EPSRC-UK) under grant EP/R513143/1.

This research was not supported by the Omnetics Connector Corporation and the authors have no relevant conflicts of interest to declare. for implantation; however, these connectors are commonly used in preclinical designs of both implants [5], [7], [8], [10], and on external head-stages [6], [11]. To justify the use of these connectors for long term preclinical implant studies implant lifetime must be investigated.

This paper presents an assembly method for Omnetics NC connectors proposed for long term preclinical testing of active implants. Assembled connectors were tested for reliability in a long-term pull-and-bend test setup and in saline solution.

\section{METHODS}

\section{A. Connector Components and Assembly}

Assembly was carried out in an ISO 14644-1 Class 6 cleanroom. Miniature 11-channel connectors (Nano 360® Plastic Circulars, NCS-11-DD and NCP-11-DD, Omnetics Connector Corporation, Minneapolis, MN, USA) were soldered to multistrand stainless steel, fluoropolymer insulated wire (AS632, Cooner Wire Company, Chatsworth, CA, USA). Solder with water soluble flux (Multicore/Loctite Hydro-X, Henkel, Hemel Hempstead, UK) was used to simplify cleaning of flux residues.

Soldered connectors (Fig. 1a) were cleaned by ultrasonication in acetone, propan-2-ol, de-ionised water, Leslie's Soup, and de-ionised water [12]. Leslie's Soup is 0.5 wt $\%$ detergent (Teepol-L, Teepol Products, Kent, UK), and $2.5 \mathrm{wt} \%$ trisodium phosphate (anhydrous, 13438, Alfa Aesar, Heysham, UK), in de-ionised water. As detailed previously cleanliness before encapsulation is essential for the survival of long-term implants [13]. Following rinsing the conductivity of the rinse solution was monitored to confirm adequate cleanliness, with conductivity less than $120 \mathrm{nS} . \mathrm{cm}^{-1}$ accepted.

Clean miniature connectors were dried at $60^{\circ} \mathrm{C}$ and encapsulated in silicone elastomer. Two different low viscosity silicones suitable for pre-clinical implants were used for encapsulation: MED-6015 (Avantor-NuSil, Radnor, PA, USA), and Kwik-Sil (World Precision Instruments, Hitchin, UK). Low viscosity encapsulant was essential to ensure the silicone reliably coats the $\mu \mathrm{m}$ scale connector parts without leaving voids for moisture condensation. MED-6015 was prepared by mixing 10:1, Part A : Part B ratio using a SpeedMixer for 1.5 minutes at $2500 \mathrm{rpm}$ (Dual Asymmetric Centrifugal Laboratory Mixer System, DAC 150 FVZ-K, Synergy Devices Ltd). MED-6015 was degassed before use at $30 \mathrm{mbar}$ centrifuge for up to $3 \mathrm{~min}$, or until bubbles were no longer visible. Kwik-Sil was supplied in self-mixing cartridges

M.H., D.J., and A.D. are with the Department of Electronic and Electrical Engineering, University College London, London, WC1E 7JE, United Kingdom.

H.L is with the Department of Medical Physics and Biomedical Engineering, University College London, London, WC1E 6BT, United Kingdom (e-mail: h.lancashire@ucl.ac.uk). 

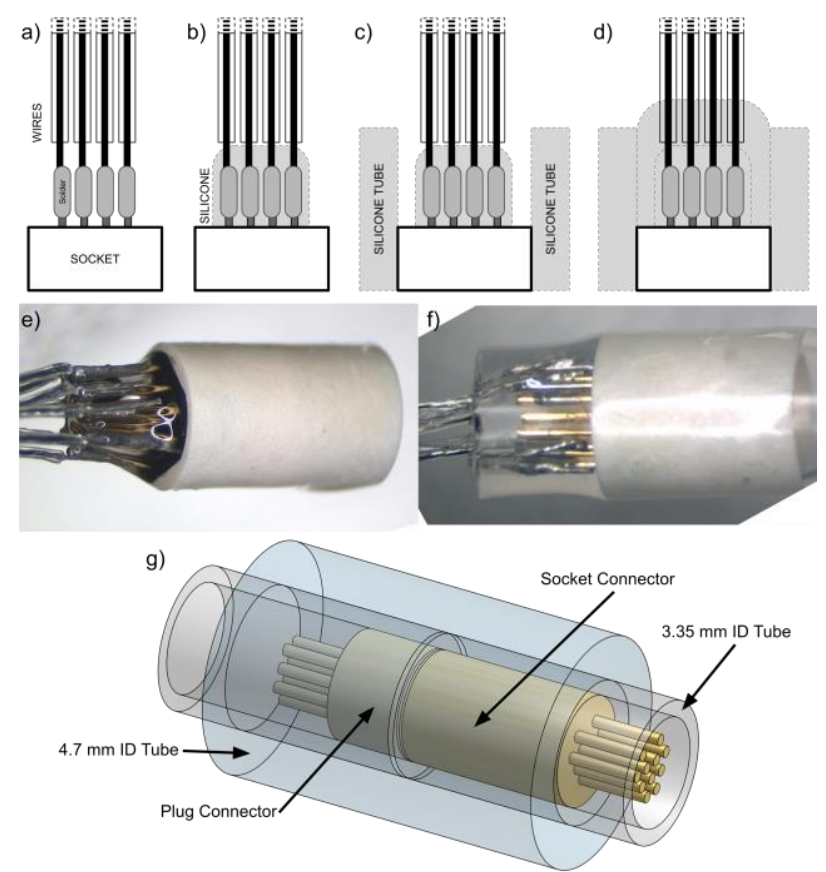

Figure 1. Encapsulation method for using Omnetics connectors in vivo (a-d, above), soldered silicone coated (e, left middle) and fully encapsulated (f, right middle) connectors, and the assembly concept for using Omnetics connectors in vivo (g, below).

and dispensed directly from the cartridge onto the part to be encapsulated.

A first layer of MED-6015 silicone elastomer was placed on the soldered pins and allowed to flow to fill voids and cover each connector (Fig. 1b and 1e). Care was taken to avoid silicone covering the outside or mating face of each connector. MED-6015 was cured at $80^{\circ} \mathrm{C}$ for 3 hours at 2.5 bar to shrink any bubbles present. Silicone tubing was placed over each connector (Fig. 1c), tubing was swelled with chloroform and press fitted to cover the connector and the soldered pins. 3.35 $\mathrm{mm}$ inner diameter (I.D.) platinum cured silicone tubing (SFM3-3350, Polymer Systems Technology, High Wycombe, UK) was chosen to fit the $3.89 \mathrm{~mm}$ outer diameter connectors tightly. MED-6015 was used to fill voids between the tubing and the previous silicone coat, and to completely cover the soldered pins (Fig. 1d). Silicone was heat cured under pressure as above. This comprised an encapsulated connector (Fig. 1d and 1f) which may be sterilized for connection during surgery.

Connections were made using a method proposed for use during surgery. $4.7 \mathrm{~mm}$ I.D. silicone tubing (SFM3-4050, Polymer Systems Technology) was passed over the encapsulated plug connector using isopropyl alcohol lubricant. Kwik-Sil was dispensed into the encapsulated socket connector and allowed to flow to coat the socket base. The 3.35 $\mathrm{mm}$ I.D. silicone tube end encapsulating the plug connector was coated with further Kwik-Sil. The connectors were mated and the $4.7 \mathrm{~mm}$ I.D. silicone tubing was passed back over both connectors to seal the joint (Fig. 1g). Manual pressure was applied to hold the connectors together for 5 minutes, sufficient for the rapid silicone curing. For use in vivo the 4.7 $\mathrm{mm}$ I.D. tubing will be replaced by a custom molded silicone sleeve with integral sutures for compression fitting.

\section{B. Long Term In-Vitro Test}

Three 11-channel plug and socket connectors were assembled for a total of $\mathrm{N}=33$ active connections.

A custom-made pull-and-bend test machine was used to apply cyclic loading to the connectors in a saline environment. Loading was applied at $1 \mathrm{~Hz}$ cycles. Phosphate buffered saline (PBS) was prepared with de-ionised water. PBS was not heated and all tests were carried out at room temperature $\left(21.4 \pm 2.4^{\circ} \mathrm{C}\right)$. Total PBS volume was maintained by addition of de-ionised water, to counteract increased concentration due to evaporation.

Contact impedance was measured at regular intervals throughout the test period using a Wayne Kerr 6500B impedance analyser. Impedance was used to investigate series contact impedance and current leakage between channels. Impedance was measured from $20 \mathrm{~Hz}$ to $30 \mathrm{MHz}$ using $1 \mathrm{~V}_{\mathrm{p}-\mathrm{p}}$ excitation.

All values are reported as mean \pm standard deviation to three significant figures unless otherwise specified.

\section{RESULTS}

Connectors were tested in PBS for 336 days. During the time in PBS, cyclic loading was applied for a total of 66 days, or over $5,700,000$ cycles. Of three connectors with 33 total contacts a single connector with 11 contacts failed under test.

\section{A. Contact Impedance}

Contact impedance for all connectors remained low for the entire test duration, with impedance of $5.87 \pm 0.10 \Omega$ at the start of testing and 6.92 $\pm 0.67 \Omega$ after 336 days (Fig. 2 and 3). Contact impedance was resistive across the frequency range of interest for neural recording and stimulation; however, inductive effects were observed at high frequencies (Fig. 2). A single connector had increased contact impedance of approximately $8 \Omega$ at 336 days, this was a different connector from the connector observed to fail.

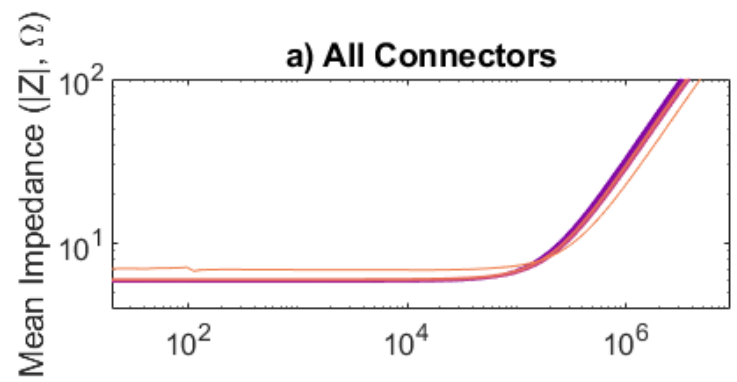

b) All Connectors

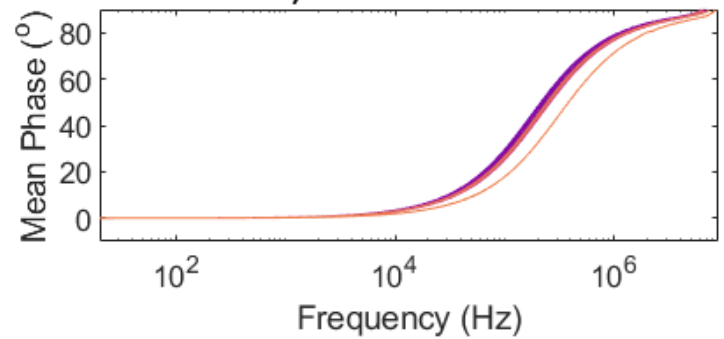

Figure 2. Bode plots of mean connector contact impedance, showing a small increase in impedance over more than 100 days in PBS. Purple / dark $=$ first measurement, orange $/$ light $=$ last measurement. 


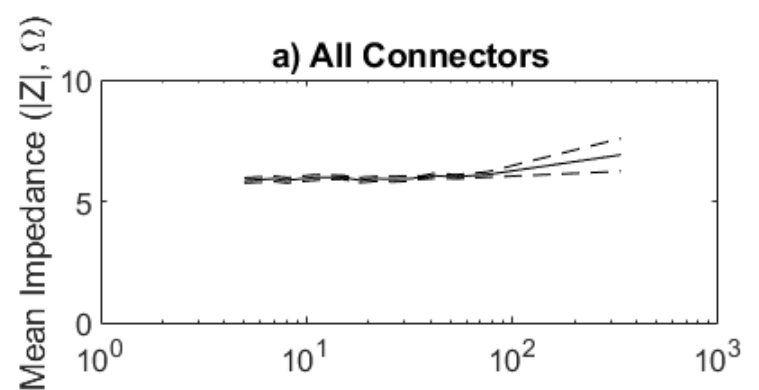

b) All Connectors

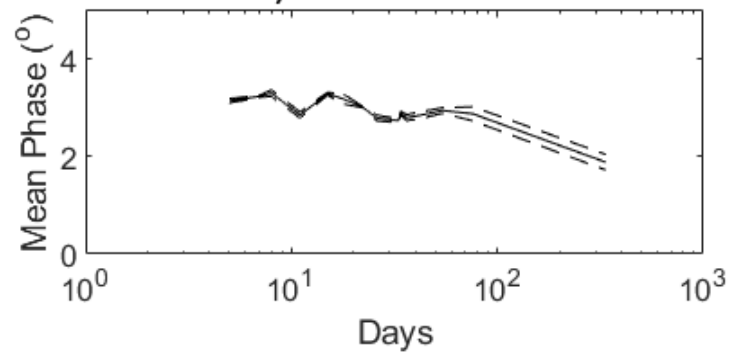

Figure 3. Change in mean connector contact impedance with time, showing a small increase in impedance over more than 100 days in PBS. Dashed lines show \pm 1 standard deviation.

\section{B. Between Channel Impedance}

The impedance between adjacent contacts was used to test for leakage currents. Impedance before testing, in dry air, was $749 \pm 79 \mathrm{k} \Omega$ at $10 \mathrm{kHz}$ for all contacts. For two connectors 10 $\mathrm{kHz}$ impedance remained $>500 \mathrm{k} \Omega(659 \pm 83 \mathrm{k} \Omega)$ at all connectors at measurement times over 336 days (Fig. 4a, 4c, and 5). For a single connector, $10 \mathrm{kHz}$ impedance declined rapidly on PBS immersion to $90.0 \pm 79.3 \mathrm{k} \Omega$ after 5 days. A minimum of $5.99 \pm 3.47 \mathrm{k} \Omega$ was observed at 76 days, and $18.8 \pm 11.4 \mathrm{k} \Omega$ after 336 days in PBS (Fig. $4 \mathrm{~b}, 4 \mathrm{~d}$, and 5 ). 10 $\mathrm{kHz}$ impedance is reported due to the $1 \mathrm{kHz}$ impedance exceeding the measurement range of the impedance analyser for surviving connectors.

The dry impedance, and impedance of surviving connectors was consistent with a $22 \mathrm{pF}$ capacitance between the channels, attributed to the measurement system and cables. This was also supported by a consistent phase of $\sim-90^{\circ}$. For the single failing connector, a leakage pathway developed. Reduced phase at lower impedances suggests the leakage path was not purely resistive.

\section{Observations}

Inspection of the failed connector showed liquid water around the connecting point, within the outer silicone tube, and localised discolouration of three solder pins on the plug connector. De-encapsulation with methyl isobutyl ketone (Sigma Aldrich) showed that of the 11 solder joints: one joint had corroded and was no longer connected; two joints showed evidence of corrosion (discoloured solder); the remaining 8 joints were in continuity with bright solder and no evidence of corrosion. The silicone encapsulant adhered well to the solder joints and to the manufacturer's encapsulation surrounding the base of the pins; however, this did not adhere to the wire insulation or to the body of the connector. A path for water ingress exists following the wire insulation to the solder joint if a void is present [13]. Corrosion could occur in the absence of a path for water ingress if a void is present at the solder
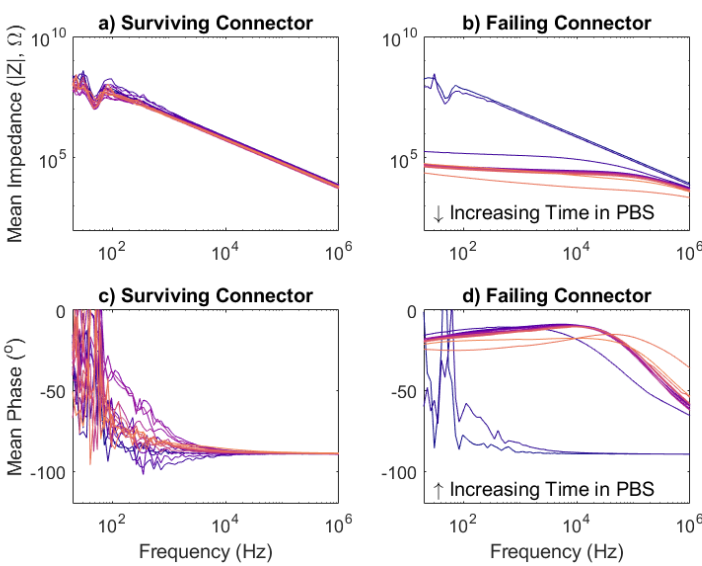

Figure 4. Bode plots of between-channel impedance, showing a connector with no observable change in impedance $(a, c)$, and a connector with an observed leakage pathway $(b, d)$. Purple $/$ dark $=$ first measurement, orange $/$ light $=$ last measurement.
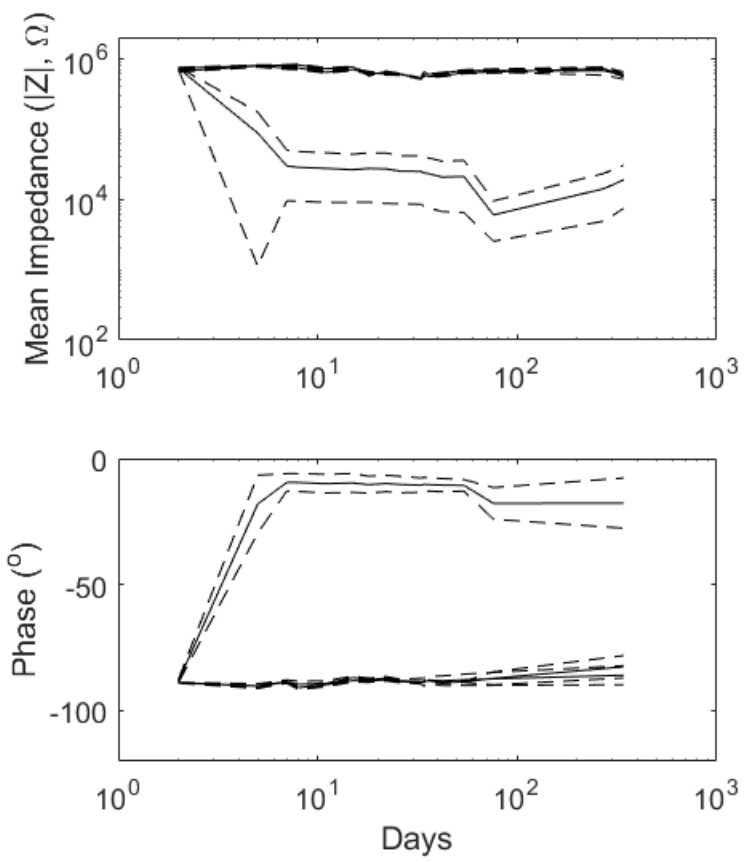

Figure 5. Change in mean between-channel impedance with time, showing a rapid decrease in impedance for one connector over approximately 1 week. Overlapping traces are for two surviving connectors. Dashed lines show \pm 1 standard deviation..

surface: local corrosion can occur in condensing moisture, creating corrosion products and driving osmosis, eventually leading to a rupturing of the silicone.

At the connecting surface Kwik-Sil silicone provided good mechanical resistance to disconnection, adhesion to the connector body was found to be poor with the silicone detaching readily once exposed. Liquid water was observed in the connecting surface on disconnection and a single socket showed evidence of corrosion. These results support the hypothesis of liquid water at the connecting surface forming a leakage path: poor adhesion to the connector body allowing a film of water to form between the silicone and the connector body. 


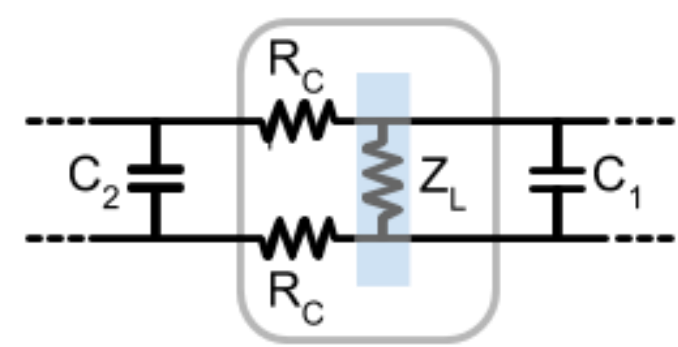

Figure 6: Proposed model of connector impedance showing two channels. $\mathrm{C}_{1}, \mathrm{C}_{2}$ is cable capacitance; $\mathrm{R}_{\mathrm{C}}$ is the contact impedance; and $\mathrm{Z}_{\mathrm{L}}$ is the moisture dependent leakage pathway. Isolation resistance is $\gg$

$\mathrm{Z}_{\mathrm{L}} \| \mathrm{C}_{1}+\mathrm{C}_{2}$ at the frequencies measured (>5G $\mathrm{G}$ ) and is not shown.

Cable resistance is not shown, small diameter cables stainless steel or platinum-iridium cables add non-negligible series resistance.

\section{DISCUSSION}

This paper has investigated the lifetime of a commercially available connector in vitro, informing the use of this connector assembly for in vivo pre-clinical testing. Two of three connectors survived over 336 days, supporting the use of silicone encapsulation of Omnetics NC connectors for preclinical investigation. However, the failure of a single connector failed under test provides important information for applying this approach. The failed connector had a shorter cable than the surviving samples. The shorter cable placed an increased load on the connector in the pull-and-bend test. This emphasizes the need for stress-relief structures, loops, or flexible cables in active implant design to minimize forces on connectors [2], [14]. Impedance change at the connector is attributed to moisture ingress into the interface between the plug and socket. The resulting impedance, with a mainly resistive path, and observations, liquid water at the connecting interface, support this hypothesis. The impedance changed progressively following moisture ingress, suggesting either a widening of the plug-socket gap, or an increase in moisture conductivity.

Corrosion at the solder joints emphasizes the need for voidfree encapsulation and sample cleanliness [13]. The investigated connectors are available pre-wired, with thermoplastic insulated copper wires. For long term implant, where adhesion of the insulation to the wires cannot be guaranteed, corrosion resistant wire materials are preferable such as the stainless steel used in this work [2].

A circuit model for the cable and connector is proposed in Fig. 6. The leakage path, $\mathrm{Z}_{\mathrm{L}}$, may be better described as a Randles equivalent circuit. Using connector geometry and the conductivity of PBS we can approximate a maximum gap size between the plug and socket. Gaps of $\sim 800 \mathrm{~nm}$ and $\sim 5 \mu \mathrm{m}$ are consistent with the impedances after 5 days and 336 days in PBS respectively. For high impedance neural electrodes, even a small volume of moisture in a connector can create a path through which a significant proportion of the intended stimulation current may leak. Different conductivity may be present due to local corrosion products, or due to moisture ingress by diffusion through silicones rather than by tracking along gaps.

The present study did not accelerate the connector aging with heating, samples were maintained at room temperature throughout. Lifetime at body temperature will be lower than observed at room temperature. The application of a pull-andbend system was used to model cyclic loads in the body, experienced by connectors near joints, adjacent to muscles with significant volume change, and placed near the lungs and heart. Further investigation is focused on increasing the number of connectors under test enable lifetime prediction.

\section{ACKNOWLEDGMENT}

The authors thank Prof. Nick Donaldson for provision of the pull-and-bend test equipment.

\section{REFERENCES}

[1] D. N. Rushton, A. M. Tromans, and N. de N. Donaldson, "A reconnectable multiway implantable connector," Med. Eng. Phys., vol. 24, no. 10, pp. 691-694, Dec. 2002, doi: 10.1016/s13504533(02)00042-5.

[2] J. Koch, M. Schuettler, C. Pasluosta, and T. Stieglitz, "Electrical connectors for neural implants: design, state of the art and future challenges of an underestimated component," J. Neural Eng., vol. 16, no. 6, p. 061002, Oct. 2019, doi: 10.1088/1741-2552/ab36df.

[3] J. E. Letechipia, P. H. Peckham, M. Gazdik, and B. Smith, "In-line lead connector for use with implanted neuroprosthesis," IEEE Trans. Biomed. Eng., vol. 38, no. 7, pp. 707-709, Jul. 1991, doi: $10.1109 / 10.83572$.

[4] P. E. K. Donaldson, "The Craggs connector; a termination for cooper cable," Med. Biol. Eng. Comput., vol. 23, no. 2, pp. 195-196, Mar. 1985, doi: 10.1007/BF02456759.

[5] M. R. MacEwan, E. R. Zellmer, J. J. Wheeler, H. Burton, and D. W. Moran, "Regenerated Sciatic Nerve Axons Stimulated through a Chronically Implanted Macro-Sieve Electrode," Front. Neurosci., vol. 10, 2016, doi: 10.3389/fnins.2016.00557.

[6] J. S. Vuong, J. J. Garrett, M. J. Connolly, A. R. York, R. E. Gross, and A. Devergnas, "Head mounted telemetry system for seizures monitoring and sleep scoring on non-human primate," J. Neurosci. Methods, vol. 346, p. 108915, Dec. 2020, doi: 10.1016/j.jneumeth.2020.108915.

[7] M. Axelsson, Q. Dang, K. Pitsillides, S. Munns, J. Hicks, and G. S. Kassab, "A novel, fully implantable, multichannel biotelemetry system for measurement of blood flow, pressure, ECG, and temperature," $J$. Appl. Physiol., vol. 102, no. 3, pp. 1220-1228, Mar. 2007, doi: 10.1152/japplphysiol.00887.2006.

[8] L. Bisoni et al., "Investigation on the hermeticity of an implantable package with 32 feedthroughs for neural prosthetic applications," in 2016 38th Annual International Conference of the IEEE Engineering in Medicine and Biology Society (EMBC), Aug. 2016, pp. 1967-1970, doi: 10.1109/EMBC.2016.7591109.

[9] "Nano 360® Circular Plastic Connectors Omnetics Connector Corporation." https://www.omnetics.com/products/micro-and-nanocirculars/nano-360-circulars-plastic (accessed Dec. 05, 2020).

[10] S. Lewis et al., "Fully Implantable Multi-Channel Measurement System for Acquisition of Muscle Activity," IEEE Trans. Instrum. Meas., vol. 62, no. 7, pp. 1972-1981, Jul. 2013, doi: 10.1109/TIM.2013.2253992.

[11] A. R. Mitz, R. Bartolo, R. C. Saunders, P. G. Browning, T. Talbot, and B. B. Averbeck, "High channel count single-unit recordings from nonhuman primate frontal cortex," J. Neurosci. Methods, vol. 289, pp. 39-47, Sep. 2017, doi: 10.1016/j.jneumeth.2017.07.001.

[12] L. Lonys et al., "Silicone rubber encapsulation for an endoscopically implantable gastrostimulator," Med. Biol. Eng. Comput., vol. 53, no. 4, pp. 319-329, Apr. 2015, doi: 10.1007/s11517-014-1236-9.

[13] A. Vanhoestenberghe and N. Donaldson, "Corrosion of silicon integrated circuits and lifetime predictions in implantable electronic devices," J. Neural Eng., vol. 10, no. 3, p. 031002, May 2013, doi: 10.1088/1741-2560/10/3/031002.

[14] P. E. K. Donaldson, "The Cooper cable: an implantable multiconductor cable for neurological prostheses," Med. Biol. Eng. Comput., vol. 21, no. 3, pp. 371-374, May 1983, doi: 10.1007/BF02478508. 